\title{
Wind profiler observations on orographic effects of typhoon wind structure modification over Taiwan $\left(120.38^{\circ} \mathrm{E}, \mathbf{2 2 . 6}^{\circ} \mathrm{N}\right)$
}

\author{
C. J. Pan ${ }^{1}$, K. Krishna Reddy ${ }^{2}$, H. C. Lai ${ }^{3}$, S. S. Yang ${ }^{1}$, and C. J. Wong ${ }^{4}$ \\ ${ }^{1}$ Institute of Space Science, National Central University, Taiwan \\ ${ }^{2}$ Department of Physics, Yogi Vemana University, Kadapa, India \\ ${ }^{3}$ Dept. of Eng. \& Manag. of Advanced Tech., Chang Jung Christian University, Taiwan \\ ${ }^{4}$ School of Physics, Universiti Sains Malaysia, Malaysia
}

Received: 4 September 2009 - Revised: 17 December 2009 - Accepted: 24 December 2009 - Published: 18 January 2010

\begin{abstract}
The interaction of the storm circulation with the Central Mountain Range (CMR) of Taiwan is studied with a wind profiler located at the leeside during the invasions of two (Kaemi (200605) and Bopha (200609)) typhoons. The moderate typhoon Kaemi upgraded from a tropical depression on 21 July 2006. It then was made landfall at 15:45 UTC on 24 July 2006 near Cheng-Kung. The weak typhoon Bopha formed at about 12:00 UTC on 5 August 2006 and also landed near Cheng-Kung at around 19:20 UTC on 8 August. A new finding from both typhoons is the vortex splitting into upper and lower parts with the two typhoons that have passed the observation site nearly. For the typhoon Kaemi, demarcation height of the upper-level vortex and lower level is at $2.8 \mathrm{~km}$ and passed the site about $3 \mathrm{~h}$ earlier than the low-level one. For the typhoon Bopha, the center of the lower-level vortex at $3.5 \mathrm{~km}$ locates to the north of the upper-level one at $5.2 \mathrm{~km}$. The re-organization of the split vortexes is found in typhoon Kaemi but not for typhoon Bopha.
\end{abstract}

Keywords. Atmospheric composition and structure (Evolution of the atmosphere)

\section{Introduction}

Typhoons are most serious natural disasters occurring in East Asia, especially in Taiwan. Since Taiwan is surrounded by oceans, it provides a unique environment for studying the orographic effects on air flow during passage of Typhoon. The Central Mountain Range (CMR) is oriented north-northeast to south-southwest with an average terrain height of about $2000 \mathrm{~m}$ (Fig. 1) and some mountain peaks

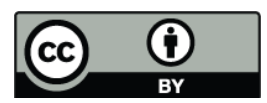

Correspondence to: C. J. Pan

(cjpan@jupiter.ss.ncu.edu.tw) reaching almost $3500 \mathrm{~m}$. Due to the high altitude and complex terrains associated with CMR, significant variations in track and intensity occur as a typhoon approaches the island (Brand and Blelloch, 1974; Wang, 1980, 1989; Yeh and Elsberry, 1993a, b) and also orographic influence on typhoon tracks is extremely complicated. The interaction of the typhoon circulation with the CMR also produces significant mesoscale variations in pressure, wind, and precipitation distribution over Taiwan. For example, the secondary low centers can be formed on the leeside of the CMR, which may develop into a new tropical cyclone, replacing the original storm (Lee et al., 2008).

Wang $(1980,1989)$ reported through observations that the center of the storm could either cross Taiwan continuously or have a discontinuous track due to the formation of a secondary low that replaces the terrain-blocked low-level center. Wang (1980) proposed that, for typhoons with a continuous track, the circulation is only modified slightly by the mountain range, while for typhoons with a discontinuous track, it is modified significantly. The Taiwan Area Mesoscale Experiment (TAMEX), jointly conducted by Taiwan and the United States during May-June 1987, is the first field experiment to focus on heavy rain problems over a mountainous island. A north-south-oriented, multi-cellular squall line observed during TAMEX was analyzed as it moved into mountainous areas (Teng et al., 2000). Since the CMR affects not only the track of a typhoon, but also its structure, several numerical modeling studies have aso addressed the effect of the terrain on typhoons crossing Taiwan (e.g., Chang, 1982; Bender et al., 1987; Yeh and Elsberry, 1993a, b; Wu and Kuo, 1999; Lin et al., 1999, 2005). Bender et al. (1987) examined the effects of different island terrains on tropical cyclones under different zonal mean flows. They suggested that the terrain induced steering flow modification was the main cause of the typhoon track deflection. In the simulation with a $10 \mathrm{~m} / \mathrm{s}$ easterly flow, a secondary surface low or

Published by Copernicus Publications on behalf of the European Geosciences Union. 


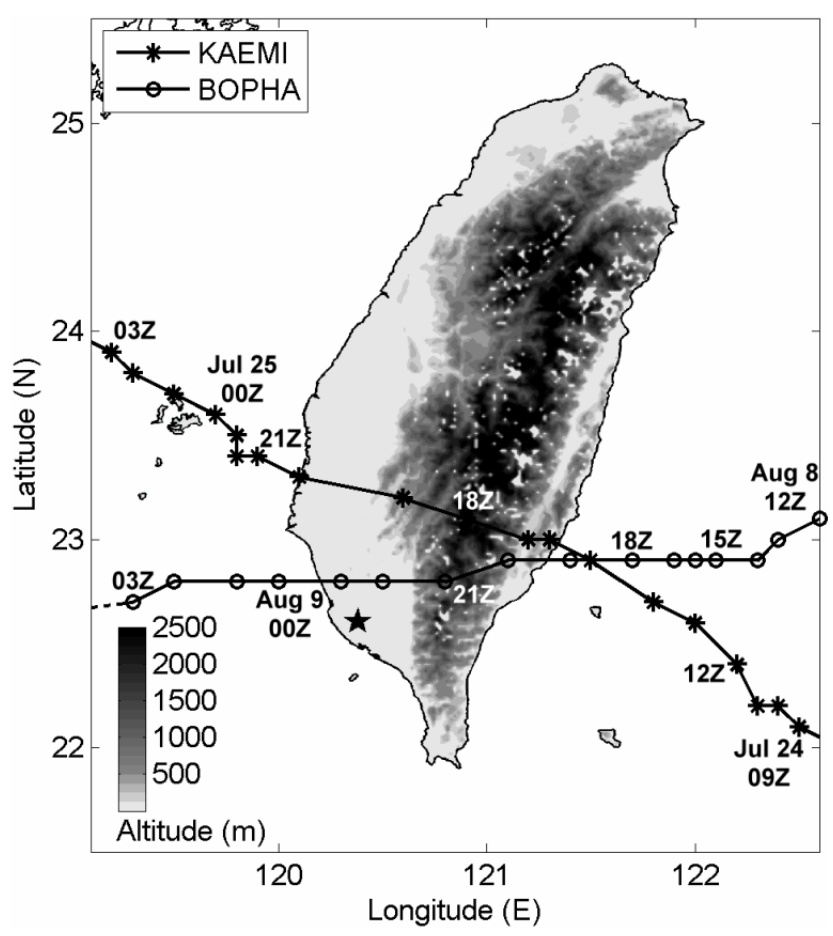

Fig. 1. Topography of Taiwan and tracks of two typhoons KAEMI (200605) and BOPHA (200609) along with the wind-profiler location indicated by star symbol.

lows formed behind the CMR. It appears that the upper-level vortex becomes detached from the original surface low and eventually couples with the secondary one. Yeh and Elsberry $(1993 a, b)$ studied the upstream track deflections of westward-moving tropical cyclones approaching the mountainous terrain of Taiwan. They showed that the amount of upstream track deflection and the continuity of the storm track across Taiwan depend on the storm's position relative to the CMR. The numerical simulations also indicate that vortex reorganization downstream may occur. For the past three decades, various observational and numerical studies on typhoons affecting Taiwan and the interaction of typhoons with the CMR have been made significant progress. Nevertheless, the high temporal and height resolution observational evidences of the storms passing over Taiwan and interaction with CMR studies are scanty. In this article, the typhoon structure affected by the CMR by using the wind profiler radar is examined in detail. For two typhoons the vortex separation due to the blocking effect at the stages of storm approaching, landing and leaving are investigated. The influences of vortex re-organization on the typhoon strength after landfall are also report in this paper.

\section{Experimental set-up}

In 2006 the DEGREWIND PCL1300 profiler was deployed at an industrial park $\left(120.38^{\circ} \mathrm{E}, 22.6^{\circ} \mathrm{N}\right)$ close to KaoHsiung city in the southern Taiwan and operated continuously almost every day in order to observe the clear and precipitation echoes in the lower atmosphere. The locations of the wind profiler are shown in the Fig. 1. The main characteristics of this profiler radar are a 1290-MHz transmitted frequency, with a $4-\mathrm{kW}$ peak pulse power, a $25-\mathrm{kHz}$ pulse repetition frequency, and a 150-m pulse length. One vertical and four oblique beams, with an off-zenith angle of 17 degrees disposed every 90 degrees in azimuth, are swinging continuously to detect the winds. The altitude coverage is from $0.235 \mathrm{~km}$ to $6.543 \mathrm{~km}$ with a $150-\mathrm{m}$ range resolution. 20 successive Doppler spectra obtained from a 128-point discrete Fourier transform are used to extract the first three moments of the atmospheric echoes. The information about the echo power intensity, radial winds as well as noise level is then deduced. We further incoherently average the derived products in the time domain to present the horizontal winds and the signal-to-noise ratio (SNR) at every $30 \mathrm{~min}$. Assuming horizontal homogeneity of the wind field (at least for the mean wind) in the volume spanned by the radar beams, one can say that the measured radial velocities are the components of the 3-dimensional wind vector along the fixed antenna directions. Afterwards a simple coordinate transformation is applied to compute the velocity vector coordinates in a Cartesian system $(U, V$ and $W)$. The wind vectors are plotted at times and heights for which a consensus was obtained among the observations in 30-min averaging period. For more details on technical information of this wind profiler, refer to Heo et al. (2003).

\section{Observational results}

The moderate typhoon Kaemi (200605) upgraded from a tropical depression on 21 July 2006. It then was made landfall at 15:45 UTC on 24 July and moved northnorthwestward to dissipate over mainland China on 25 July. We reported in this paper, wind profiler observations starting from 09:00 to 24:00 UTC of 24 July that covered the time periods of typhoon approaching (from 09:00 to 15:44 UTC), landing (from 15:45 to 20:05) and leaving (from 20:06 to 24:00 UTC) Taiwan, respectively. The weak typhoon Bopha (200609) formed at about 12:00 UTC of 5 August 2006 and also landed around 19:20 UTC on 8 August. It then proceeded westward until it decayed over the sea of Taiwan banks on 9 August 2006. Typhoon Bopha approached from 12:00 to 19:20 UTC, landfall from 19:21 to 23:20 UTC and moved from 23:21 on 8 August to 03:00 of 9 August 2006 . The typhoon Kaemi and Bopha (200609) crossed the CMR and passed from the north of the observation site with the closest distance of about $60 \mathrm{~km}$ and $20 \mathrm{~km}$, respectively. 
1

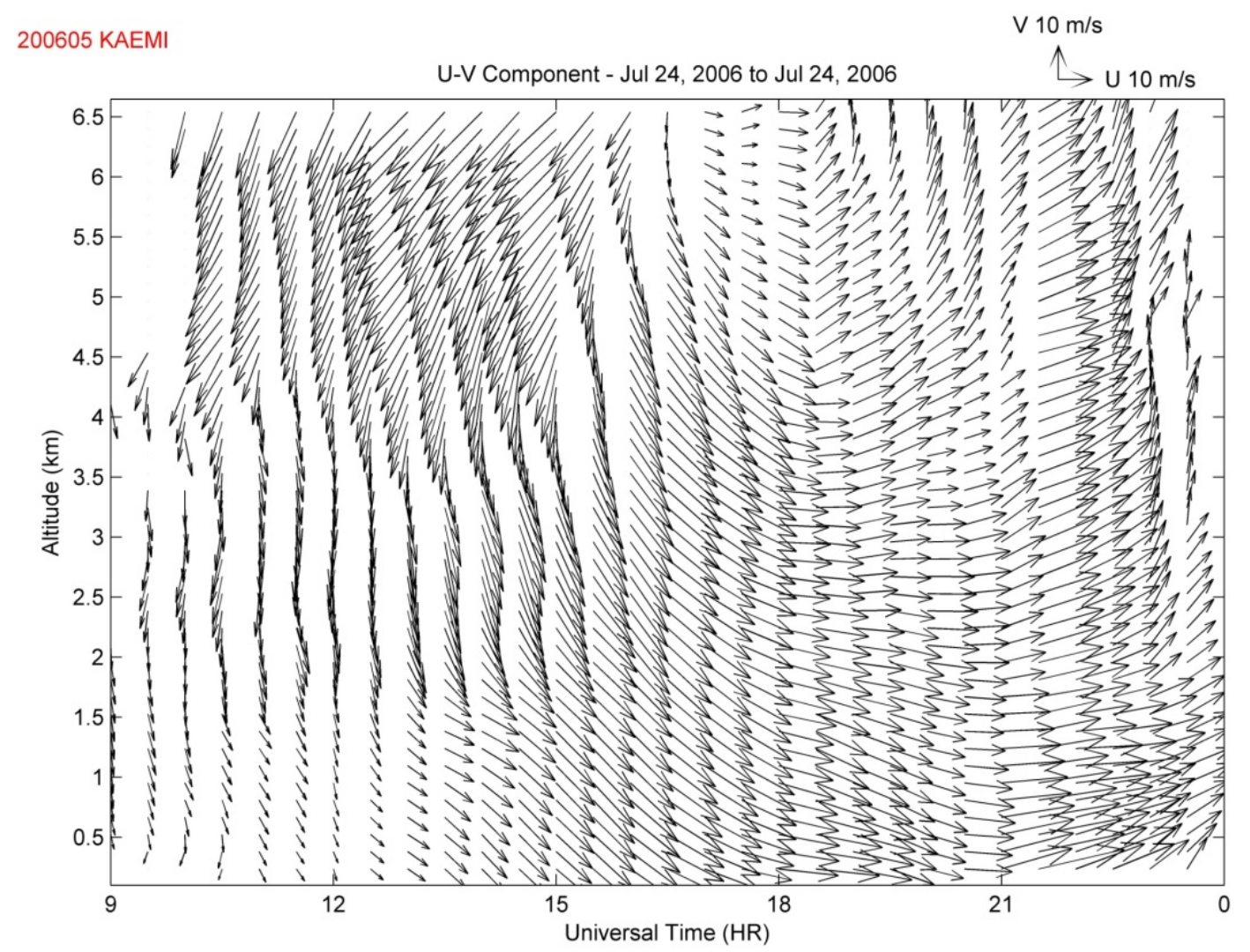

Fig. 2. Altitude-time profile of the Horizontal wind observed during the passage of Typhoon Kaemi.

Figure 1 shows the topography of Taiwan, the tracks (the best track analysis provided by the Central Weather Bureau (CWB) of Taiwan) of two typhoons and the wind profiler radar locations. Two typhoons, namely, Kaemi (black line with star) and Bopha (black line with open circle) invaded Taiwan in the summer of 2006. Due to the high altitude and complicated mesoscale topography associated with Taiwan's CMR, which has an average elevation higher than $2500 \mathrm{~m}$ and a dimension of $300 \mathrm{~km} \times 100 \mathrm{~km}$, significant variations in track and intensity occur as both typhoons approaches the island. From the typhoon best track analysis provided by the CWB suggests that clear turning of wind field from northeasterly to south-westerly associated with the passage of typhoon is anticipated from the wind profiler observations.

Figure 2 denotes the time- height distributions of the zonal $(U)$ - meridional $(V)$ wind component observed on 24 July 2006 between 09:00 UTC and 24:00 UTC by the wind profiler during the typhoon Kaemi invasion. As we can see, the northeasterly wind was dominated above $3.5 \mathrm{~km}$ from 10:30 to 16:00 UTC but the northerly to northwesterly winds took place below $3.5 \mathrm{~km}$ while the typhoon center was about $200 \mathrm{~km}$ to $100 \mathrm{~km}$ away from the observation site. This demarcation of different wind directions at above and below $3.5 \mathrm{~km}$ altitude implies that the blocking effect of the CMR is significant below $3.5 \mathrm{~km}$ altitude even though the typhoon was still $200 \mathrm{~km}$ to the east of CMR and the dominance of blocking effect was prevailed till 16:00 UTC of 24 July when the typhoon Kaemi was about to land. From about 16:30 UTC of 24 July, the horizontal winds changed anticlockwise to northwesterly and continued in this direction till 18:30 UTC, when the typhoon center was closest $(69 \mathrm{~km})$ to the profiler, then changed to southwesterly direction from 18:30 UTC. It is interesting to notify that no significant wind changes in the vertical direction after the typhoon landed as those detected before 16:00 UTC. This may signify a reorganization of typhoon wind structure and weakening of blocking effect of the CMR after the typhoon center made landfall.

Figure 3 shows the time- height distributions of horizontal winds observed from 12:00 UTC on 8 August 2006 to 03:00 UTC of 9 August 2006. Typhoon Bopha approached from 12:00 to 19:20 UTC, made landfall from about 19:00 to 23:00 UTC and moved from 23:30 UTC of 8 August to 03:00 UTC of 9 August. The weak typhoon Bopha formed at about 12:00 UTC of 5 August 2006 and also landed near Cheng-Kung at around 19:30 UTC of 8 August. It then proceeded westward until it decayed over the sea of Taiwan banks on 9 August. Although the horizontal winds are more 


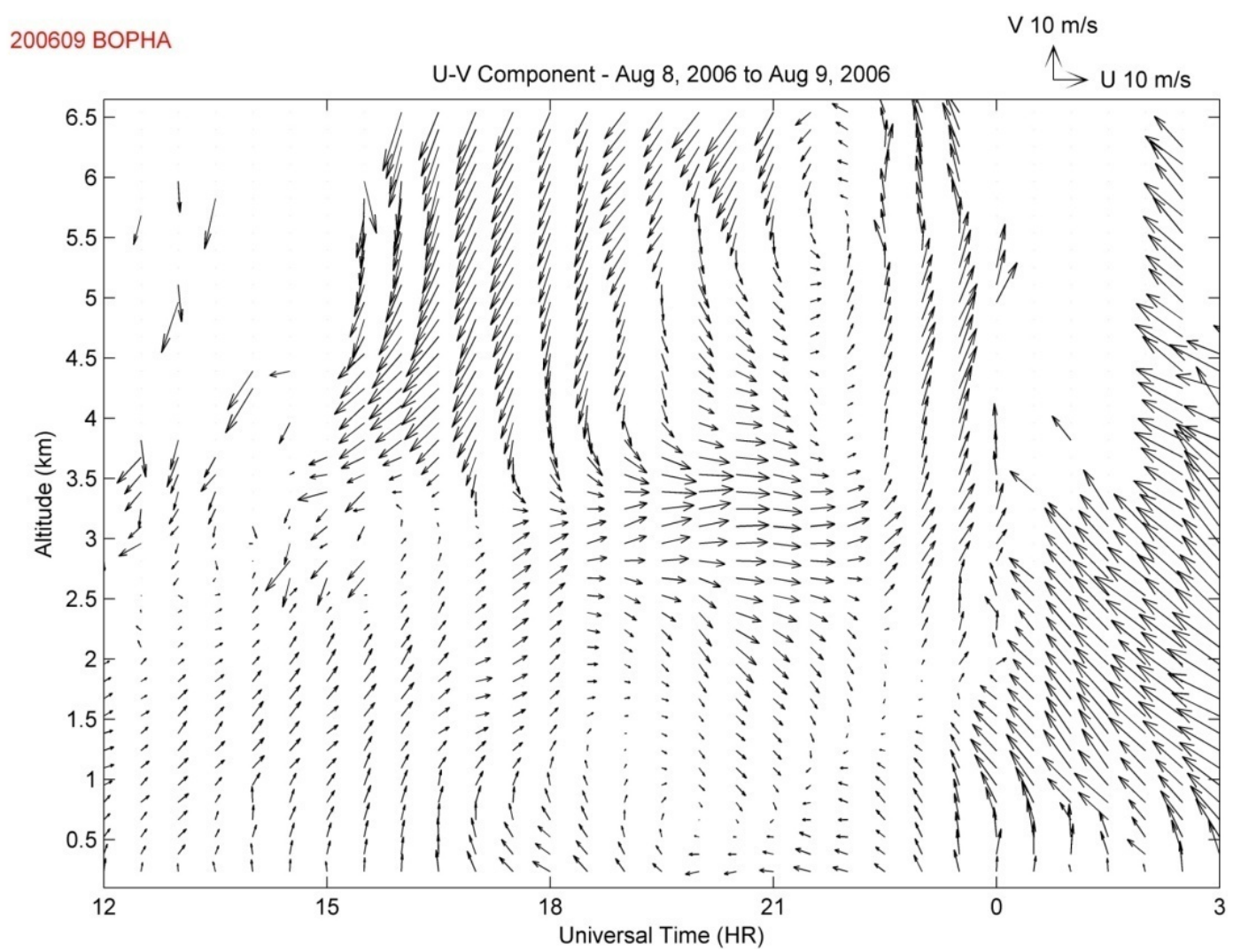

Fig. 3. Altitude-time profile of the Horizontal wind observed during the passage of Typhoon Bopha.

complicated than those of Typhoon Kaemi, it is clearly seen that the wind direction is northeasterly above $3.5 \mathrm{~km}$ altitude and mainly southwesterly below $3.5 \mathrm{~km}$ altitude from about 15:30 to 19:00 UTC of 8 August. It is again evident from this observation that the blocking effect of CMR to the wind field is dominant below $3.5 \mathrm{~km}$ altitude.

The center of typhoon Bopha landed at 19:30 UTC and was estimated to have passed north of the wind profiler at around 22:30 UTC with the minimum distance of about $22 \mathrm{~km}$. It further weakened and passed over Taiwan at about 23:30 UTC of 8 August. During 17:00 UTC to 23:00 UTC, the winds detected near surface are mainly from the east, which is consistent with the report of the Kao-Hsiung surface station of the Central Weather Bureau (not shown here). Those easterly winds seem "lift-up" the southwesterly winds below $3.5 \mathrm{~km}$ altitude where the blocking effect of CMR takes place before the typhoon landing. With the westerly jet-like winds centered at about $3 \mathrm{~km}$ altitude during 18:00 UTC to 22:00 UTC, a rotor-look structure with core at $700 \mathrm{~m}$ altitude and above is notified. A clear turning of wind fields as the typhoon reaches the closest distance of wind profiler around 22:30 UTC is again observed. Nevertheless, this feature only takes place at the region above about $2 \mathrm{~km}$ altitude. The wind structure seems not systematically organized after the typhoon center climbs over the CMR.
Figure 4 shows the wind profiler observations of typhoon Kaemi and Bopha during the periods of typhoon approaching, landing and leaving Taiwan, respectively. The heighttime distributions of the signal-to-noise ratio (the top panel) and the zonal and meridional components of the winds (the second and the third panels) observed by the wind profiler are shown in Fig. 4. The horizontal distributions of precipitation associated with typhoon Kaemi and Bopha eyewall and rain bands (as measured by the signal-to-noise ratio shown in the upper panel of Fig. 4) show a pronounced bright band at around $5 \mathrm{~km}$ altitude. Since both are characterized by a bright band, these precipitation types are stratiform ones (Williams et al., 1995). After the landfall of typhoon center, the bright bands are occasionally seen that indicates convective type of rainfall becomes dominant within the eyewall. No data are reported within the distance of $60 \mathrm{~km}$ and $20 \mathrm{~km}$, respectively, for Kaemi and Bopha and they are beyond the observational area.

From about 16:30 UTC of 24 July, the horizontal winds changed anti-clockwise to northwesterly and continued in this direction till 18:30 UTC, when the typhoon center was closest $(69 \mathrm{~km})$ to the profiler, then changed to southwesterly direction from 18:30 UTC. It is interested to notify that no significant wind changes in the vertical direction after the typhoon landed as those detected before 16:00 UTC. This may signify a re-organization of typhoon wind structure and 

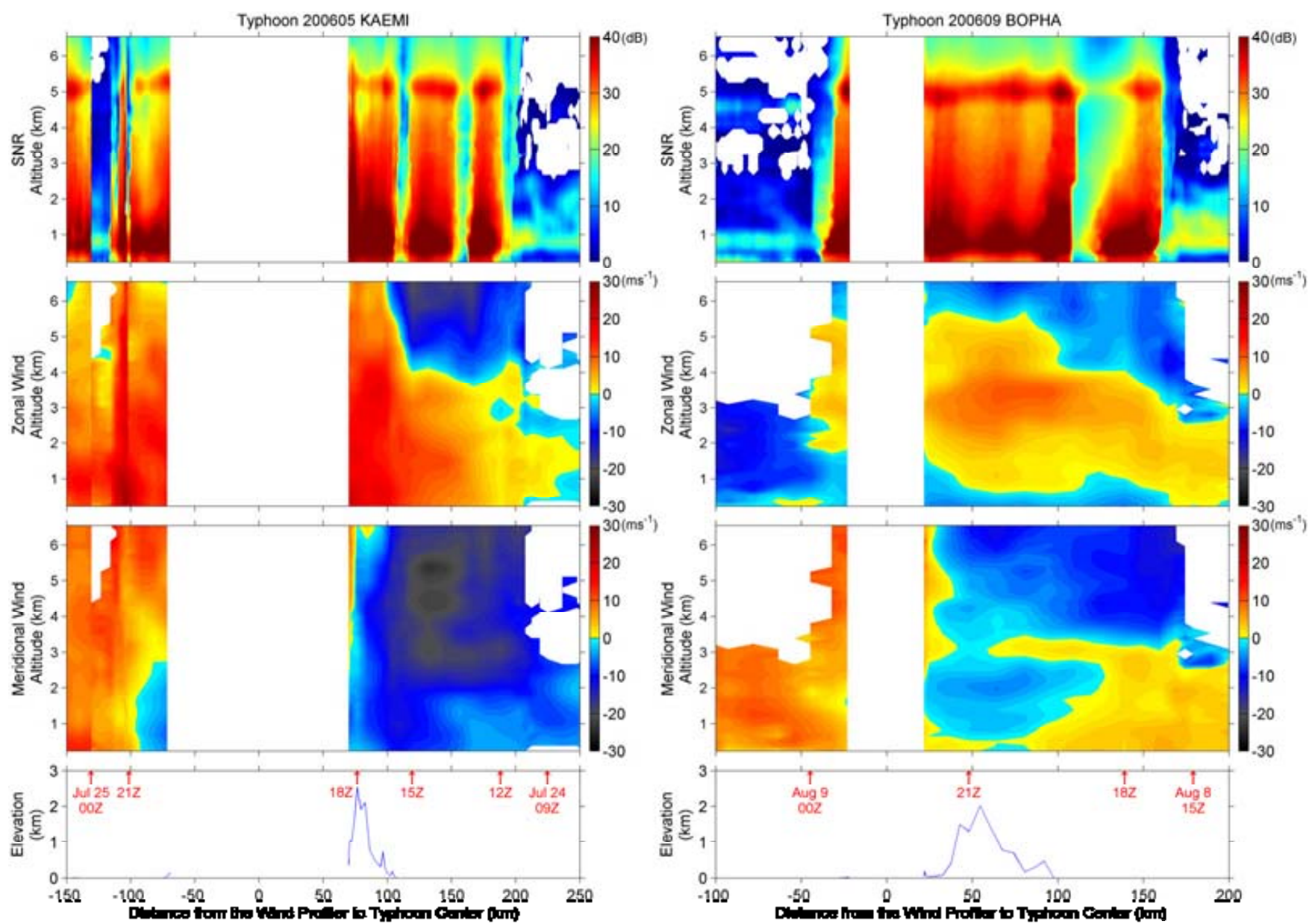

Fig. 4. The height-time distributions of the signal-to-noise ratio (the top panel) and the zonal and meridional components of the winds (the second and the third panels). The bottom panel shows the distances between the wind profiler and the Typhoon center with respect to the progress of time and orography of the CMR. The left and the right panel represent the typhoon Kaemi and Bopha, respectively.

weakening of blocking effect of the CMR after the typhoon center made landfall.

Though the horizontal winds associated with typhoon Bopha above $3.5 \mathrm{~km}$ behaved, initially, similarly as typhoon Kaemi associated winds, the wind field became very complicated with the progress of time. For instance, the horizontal winds (belong to typhoon leaving period) after 23:30 UTC behaved irregularly until the end of observations (03:00 UTC). On the other hand, no continuous rain band is observed from 23:30 UTC of 8 August 2006 to 03:00 UTC of 9 August 2006.

The distances between the wind profiler and the typhoon center are presented in Fig. 4 (bottom panel) with respect to the progress of time and orography of the CMR. The positive and negative values of radius correspond to the period when the storm approaches (in front side of typhoon) and leaves (in rear side of typhoon) to the observation site, respectively. For the zonal wind display, a significant wind shear takes place at about $3 \sim 4 \mathrm{~km}$ altitude for both typhoons from the outer band reaches the site to the storm is about to land. The easterly winds dominate aloft since the cyclone is still to the southeast of Taiwan and there is no mountain blocking above about $3.5 \mathrm{~km}$ altitude. On the other hand, westerly flow presents in the zonal component due to the wind deflection from the terrain blocking below about $3.5 \mathrm{~km}$ (Pan et al., 2008). After the landfall of typhoon Kaemi, the easterly flow is replaced by the westerly wind from below and no wind shear is seen in the zonal component of the winds up to $6.5 \mathrm{~km}$ altitude, the upper bound of the observation. The westerly wind lasts till the end of observation.

Around the period of typhoon Bopha landing, the zonal wind shear appears at higher region of about $5.2 \mathrm{~km}$ altitude with easterly at above and westerly at below. As the storm is passing across the CMR, the mountain blocking becomes insignificant. The wind shear at $5.2 \mathrm{~km}$ is caused by other meteorological mechanism and will be discussed later. Meanwhile, the near surface easterly winds of typhoon Bopha that last as long as the end of the observation may be due to the 
interference from other weather systems that is beyond this investigation.

The meridional component of the winds show a different features for these two typhoons. Without mountain blocking, northerly component is observed at above $3.5 \mathrm{~km}$ altitude for both typhoons since their outer circulations approach. As the storm centers move close to the wind profiler, southerly winds become dominant gradually to all heights due to the turning/veering of cyclone wind field. The southerly wind is noted below about $3.5 \mathrm{~km}$ altitude when typhoon Bopha is approaching the island.

\section{Discussion}

Mountain blocking causes the flow field associated with a typhoon impinges the CMR with a low-level flow passing around, but upper-level flow crossing over, the barrier. It may even appear that the upper-level vortex get detached from the original surface low and moves separately after typhoon landing. On the other hand, in the regions "shadowed" by the significant blocking effect, the wind fields behave diversely depending on different typhoon. It is interesting to study the features of the airflow of the upper- and lower-level vortex at different stages during the typhoon crosses the CMR.

Without blocking from the mountain ranges, northeasterly flow tends to dominate the regions at above $3 \sim 3.5 \mathrm{~km}$ altitude for those two typhoons presented in this study. With easterly above and westerly below $3 \sim 3.5 \mathrm{~km}$, it is easy to allocate the period when the blocking effect takes place by verifying the vertical wind shear of the zonal winds. This phenomenon is noted since the typhoon's outer bands reach the observation site and gets weakened when the typhoon center is about to land. Dhaka et al. (2003) investigated the typhoon Orchid (9426) passed over the MU radar on 29-30 September 1994. They found that the typhoon, the mesoa-scale wind field is characterized by the low-level cyclonic wind with the maximum and outflow regions tilted outward with height.

Due to the terrain blocking, during 10:00 UTC to 15:00 UTC of 24 July when typhoon Kaemi is approaching, the lower-level mountain-induced winds become northwesterly owing to be along with the topography. After the typhoon makes landfall, the blocking effect tends to become weaker and the westerly flows replace the easterly winds that originally dominate above $3.5 \mathrm{~km}$ altitude. As the storm center is approaching the nearest place to the north of the observation site at about 18:00 UTC of 24 July, the winds turn from north-westerly to south-westerly above $2.8 \mathrm{~km}$ altitude. This corresponds to a westward-moving typhoon with the winds in the third (southwest) quadrant followed by those in the fourth (southeast) quadrant of the cyclone circulation. It is worth noticing that the lower-level winds changing are delayed for about $3 \mathrm{~h}$ until 21:00 UTC, which is clearly identified from the meridional profile of the winds. Although it is difficult to detect the typhoon circulation by one wind profiler only, the incoherency of airflow in the vertical profile may indicate two vortices above the wind profiler. The reversion from northwesterly to southwesterly at the upper region (above $2.8 \mathrm{~km}$ ) occurs $3 \mathrm{~h}$ earlier than that in the lower region. We may reasonably assume that the upper center moves faster or occurs earlier than the lower one. Typhoon Bopha shows more complicated flow deflection when it approaches the CMR. Although, like the typhoon Kaemi, northeasterly above $3.5 \mathrm{~km}$ altitude is significant due to terrain blocking from about 15:00 UTC to 20:00 UTC of 8 August, southwesterly dominates at the lower altitudes. It is clearly seen that the southerly flows are confined below the altitudes of about $3 \mathrm{~km}$ from the meridional profile of the winds.

As reported earlier research studies (Wang, 1989; Tsay, 1994), a secondary circulation may form over the west coast as the low-level flow of the typhoon passes around the mountain ranges from the northern side of Taiwan and deflects toward the north as it impinges the CMR. For the observation of typhoon Bopha, a secondary vortex, extending up to at least $3 \mathrm{~km}$ altitudes, may induce southwesterly flows as it locates to the northwest of the wind profiler. Before the storm makes landfall, we presume that the terrain-induced flow deflections are mainly confined to the lower levels to form a cyclonic circulation on the leeside of the CMR. After the storm center reaches the island, westerly extends aloft up to about $5.2 \mathrm{~km}$ altitude although easterly flows are noted at above. This indicates the weakening of mountain blocking after typhoon landing as those shows in these two storms. During the westbound typhoon Bopha passing over the mountain ranges, from 20:00 UTC to 23:00 UTC of 8 August, the winds above $5.2 \mathrm{~km}$ change from north-easterly to southeasterly and north-westerly to south-westerly at below. For the flow of the upper-level one, it changes from in the second (northwest) to the first (northeast) quadrant of the cyclone. On the other hand, the lower-level part is from the third to the fourth quadrant. The center of the upper-level circulation locates to the south of the wind profiler since the first and the second quadrant winds are detected. On the contrast, the low-level center is to the north of the site. This may be due to the original storm has split into two vortices to cross the mountainous terrain, separately. This needs further detailed investigations.

Finally, using one wind profiler at a fixed point is impossible to observe the evolution of the typhoon reorganization although vortex splitting is reported in this study. The winds become re-organized above the observation site for the case of typhoon Kaemi after 21:00 UTC of 24 July. The winds re-unify may due to the upper-level vortex extends downward or just because the winds are all in the fourth quadrant of the storm. On the other hand, the upper- and the lower level vortices of typhoon Bopha were keeping separately even when it had moved away from the CMR, since the horizontal wind shear at about $5.2 \mathrm{~km}$ was still lasting. The storm was weakened due to the inconsistency of the vortex 
centers that caused a vertical tilting/distorting of the typhoon system. This is ascertained by the storm dissipated just $6 \mathrm{~h}$ later over the sea after its center moved away from Taiwan.

\section{Conclusion}

The observational data collected from a wind profiler is used to investigate the CMR affect both the airflow and storms impinging on the island significantly since two-thirds of the landmass of Taiwan is covered by rugged terrain. This article focuses mainly on the vertical profile of the horizontal wind structure analysis and features of two typhoons during different phases of typhoon invading Taiwan Island. In this study, it is commonly seen that the outer circulation of the storm interacts with the CMR that turns northeasterly winds above about $3.5 \mathrm{~km}$ in altitude and deflects with the blocking effect at lower altitudes. For the first-time, the phenomena of two split vortices associated with the typhoon due to the terrain blocking for different events, are reported.

The wind profiler observations (with good temporal and vertical resolutions) of horizontal winds provide finer details of the typhoon-orography interactions. From the observations, it is also evident that very complicated wind structures due to the interaction of the typhoon with the CMR. Those information shall be important for verification of model simulations and also improved models can be developed for typhoon research and prediction.

Acknowledgements. This wind profiler is operated by the Environmental Protection Administration (EPA) of Taiwan. C. J. Pan is supported by the NSC of Taiwan, R.O.C. through grant NSC 952111-M-008-012-MY3.

Topical Editor F. D'Andrea thanks S. Dhaka for his help in evaluating this paper.

\section{References}

Bender, M. A., Tuleya, R. E., and Kurihara, Y.: A numerical study of the effect of an island terrain on tropical cyclones, Mon. Weather Rev., 115, 130-155, 1987.

Brand, S. and Blelloch, J. W.: Changes in the characteristics of typhoons crossing the island of Taiwan, Mon. Weather Rev., 102, 708-713, 1974.

Chang, S. W.-J.: The orographic effects induced by an island mountain range on propagating tropical cyclones, Mon. Weather Rev., 110, 1255-1270, 1982.
Dhaka, S. K., Takahashi, M., Shibagaki, Y., Yamanaka, M. D., and Fukao, S.: Gravity wave generation in the lower stratosphere due to passage of the typhoon 9426 (Orchid) observed by the MU radar at Shigaraki $\left(34.85^{\circ} \mathrm{N}, 136.10^{\circ} \mathrm{E}\right)$, J. Geophys. Res., 108(D19), 4595, doi:10.1029/2003JD003489, 2003.

Heo, B.-H., Jacoby-Koaly, S., Kim, K.-E., Campistron, B., Benech, B., and Jung, E.-S.: Use of the Doppler spectral width to improve the estimation of the convective boundary layer height from UHF wind profiler observations, J. Atmos. Oceanic Technol., 20, 408424, 2003.

Lee.C.-S., Liu, Y.-C., and Chien, F.-C.: The secondary low and heavy rainfall associated with typhoon Mindulle (2004), Mon. Weather Rev., 136, 1160-1283, 2008.

Lin, Y.-L., Han, J., Hamilton, D. W., and Huang, C.-Y.: Orographic influence on a drifting cyclone, J. Atmos. Sci., 56, 534-562, 1999.

Lin, Y.-L., Chen, S.-Y., Hill, C. M., and Huang, C.-Y.: Control parameters for the influence of a mesoscale mountain range on cyclone track continuity and deflection, J. Atmos. Sci., 62, 18491866, 2005.

Pan, C. J., Lai, H. C., Yang, S. S., Reddy, K. K., and Chang, S.-C.: Wind profiler radar investigation on typhoonorography interaction, Geophys. Res. Lett., 35, L24812, doi:10.1029/2008GL036368, 2008.

Teng, J.-H., Chen, C.-S., Wang, T.-C. C., and Chen, Y.-L.: Orographic Effects on a Squall Line System over Taiwan, Mon. Weather Rev., 128, 1123-1138, doi:10.1175/1520-0493, 2000.

Tsay, C.-Y.: Orographic effects on the structure of Typhoons: Analyses of two typhoons crossing Taiwan, Terr. Atmos. Ocean. Sci., 5(2), 313-333, 1994.

Wang, S.-T.: Prediction of the movement and strength of typhoons in Taiwan and its vicinity, Research Rep. 108, 100 pp., National Science Council of Taiwan, Taipei, Taiwan, 1980.

Wang, S.-T.: Observational analysis of the orographically induced disturbances during TAMEX, Workshop on TAMEX Preliminary Scientific Results, 279-286, Taipei, Taiwan, 1989.

Williams, C. R., Ecklund, W. L., and Gage, K. S.: Classification of precipitating clouds in the tropics using $915-\mathrm{MHz}$ wind profilers, J. Atmos. Oceanic Technol., 12, 996-1012, 1995.

Wu, C.-C. and Kuo, Y.-H.: Typhoon affecting Taiwan: current understanding and future challenges, B. Am. Meteorol. Soc., 80, 67-80, 1999.

Yeh, T.-C. and Elsberry, R. L.: Interaction of typhoons with the Taiwan topography. Part I: Upstream track deflection, Mon. Weather Rev., 121, 3193-3212, 1993a.

Yeh, T.-C. and Elsberry, R. L.: Interaction of typhoons with the Taiwan topography. Part II: Continuous and discontinuous tracks across the island, Mon. Weather Rev., 121, 3213-3233, 1993b. 